\title{
Treatment of Bleeding Secondary to Gastric Metastases from Renal Cell Carcinoma Primary
}

\author{
Katherine M Guest*, George J Joy, Robin Som and Rajab Kerwat \\ Department of General Surgery, Queen Elizabeth Hospital, Stadium Road, London, UK
}

\begin{abstract}
Gastric metastases from primary renal cell carcinoma (RCC) are uncommon, but not as rare as once thought. These metastases frequently present with upper gastrointestinal (UGI) bleeding. We report two such cases, and utilising lessons learnt from them, and from a literature review, propose a model of treatment for UGI bleeding secondary to metastases from RCC primaries.

Both patients presented with clinically significant UGI bleeding secondary to RCC metastasis to the stomach. A literature search was conducted and a qualitative review of the published case reports and studies were undertaken.

The two cases were discussed in a multi-disciplinary setting to plan management. One patient underwent gastric wedge resection; the second patient received palliative radiotherapy. Cessation of bleeding was achieved in both cases. A total of 48 cases were identified from the literature search. The reports indicate that surgery for gastric metastases has favourable outcomes in patients who do not have concurrent metastases. Palliative radiotherapy in this setting has not previously been described. A model of how these patients could be managed was subsequently constructed; the key question to answer is how disseminated the disease is.

Treatment modalities are still debated and should be discussed on a case-by-case basis. However, the literature suggests that surgical intervention has good therapeutic and prognostic benefit in patients with isolated metastatic disease to the stomach. For those with widespread metastases, there are several management options available. We advise that radiotherapy should also be considered as an option in the management of patients with bleeding lesions and concurrent metastatic disease.
\end{abstract}

Keywords: Renal cell carcinoma; Metastasis; Stomach; Gastrointestinal bleeding

\section{Introduction}

Gastric metastases from primary renal cell carcinoma (RCC) are considered rare. However, there are nearly 50 cases described in the literature. We present two cases of patients presenting with clinically significant bleeding from gastric RCC metastasis; utilising lessons learnt from these cases, and from a literature review, we propose a model of treatment for gastrointestinal (GI) bleeding secondary to metastases from RCC.

\section{Case 1}

A 68-year-old Caucasian lady (Ms DA) was referred to the emergency department (ED) by her general practitioner following a collapse. She complained of fatigue, anorexia and weight loss of around 1.5 stone over 6 months. Blood tests revealed a microcytic anaemia (haemoglobin $=51$ grams/litre, mean corpuscular volume $(\mathrm{MCV})=$ 71 femtolitres).

Past medical history was significant for left-sided clear cell renal cell carcinoma 21 years prior to this presentation, for which she underwent a nephrectomy. Her drug history included levothyroxine, aspirin and prochlorperazine. Ms DA lived with her partner, was a non-smoker and consumed no alcohol. Physical examination at the time of presentation did not reveal anything of note.

Initial treatment involved resuscitation and transfusion of 3 units of packed red cells and an infusion of vitamin B complex. Her haemoglobin levels returned to normal parameters following this.

A computerised tomography (CT) scan showed an elevated soft tissue lesion arising from the posterior wall of the central stomach with no evidence of metastatic disease.
Ms DA subsequently underwent a gastroscopy which showed 2 ulcerated, sessile polyps, the largest measuring 20 millimetres, and a submucosal polyp on the greater curvature of the stomach. All lesions were biopsied. A colonoscopy was attempted but was limited by melaena.

A positron emission tomography CT scan and a nuclear medicine bone scan were performed, neither of which showed metastatic disease.

Histological examination of the biopsies showed clear cell RCC metastasis. Her case was discussed at a multi-disciplinary meeting (MDM), where it was decided that a laparoscopic gastric wedge resection would be most appropriate for her. This procedure was performed successfully without post-operative complications.

Histology of the resected specimen showed a well circumscribed 15 millimetre nodule in the submucosa with ulceration into the mucosa, no spread into stomach muscle and clear surgical margins.

Since this, Ms DA has annual surveillance gastroscopies which have not shown any evidence of recurrence (Figure 1).

*Corresponding author: KatherineMGuest, QueenElizabeth Hospital, Stadium Road, Woolwich, London, UK, SE184QH, Tel: +447454813404; Fax:+442088364590; E-mail: katherineguest@doctors.org.uk

Received October 20, 2014; Accepted October 24, 2015; Published October 31 2015

Citation: Guest KM, Joy GJ, Som R, Kerwat R. Treatment of Bleeding Secondary to Gastric Metastases from Renal Cell Carcinoma Primary. Journal of Surgery [Jurnalul de chirurgie]. 2015; 11(4): 153-159 DOI:10.7438/1584-9341-11-4-7

Copyright: ( 2015 Guest KM, et al. This is an open-access article distributed under the terms of the Creative Commons Attribution License, which permits unrestricted use, distribution, and reproduction in any medium, provided the original author and source are credited. 


\section{Case 2}

A 73-year-old Asian lady (Mrs HB) presented to the ED with a one day history of per rectal bleeding, including both fresh blood and melaena. There were no associated symptoms and examination was unremarkable.

Serological investigations confirmed a microcytic anaemia, with haemoglobin $=82$ grams/litre, and $\mathrm{MCV}=77.3$ femtolitres. She had already been receiving iron supplementation for this.

In 2005, she underwent left-sided nephrectomy for clear cell RCC. Surveillance CT scan that year had shown nodules in the nephrectomy bed as well as pulmonary spread. She received Sunitinib for this.

During her admission she required repeated transfusions of packed red cells for continuous per rectal bleeding and persistent anaemia.

A gastroscopy showed a distal gastric lesion in the greater curvature of the stomach with ulcerated overlying mucosa and clot. Biopsies of the lesion were taken which confirmed clear cell RCC metastasis.

Mrs HB's case was discussed in a MDM, where it was felt surgical intervention would not be appropriate given the presence of multiple metastases. Palliative radiotherapy was thought to be the best course of treatment in view of her ongoing bleeding. Thus, she underwent a repeat gastroscopy to reassess the lesion and endoscopically place marking clips for focused palliative radiotherapy. Cessation of bleeding was successfully achieved (Figure 2).

\section{Literature Review}

Renal cell carcinomas account for nearly $2 \%$ of cancers worldwide and are associated with high rates of metastases, with these often occurring several months after curative treatment [1,2]. Metastases originating from RCCs are most commonly found in the lungs, brain, breast and bones and are of clear cell histology [3-8].

\section{Method}

A review of the current literature on metastatic renal cell carcinoma to the stomach was performed using PubMed. The search terms were: "renal cell carcinoma stomach" + "renal cell carcinoma metastasis stomach". A total of 48 cases were identified (Table I).

\section{Demographics and clinical characteristics}

The majority of patients were male (67\%) with a mean age of 67 years (range $45-83$ years). The mean interval post-nephrectomy was 6.9 years. This implies a significant delay in the development of gastric metastases following curative treatment. Melaena was the most frequently reported presenting complaint (46\%).

\section{Tumour characteristics}

Clear cell histology was evident in all cases which reported a histopathological diagnosis. Over two thirds of patients had single lesions with the appearance of polyps, ulcers or tumours. The majority of lesions were located in the body of the stomach (63\%).

Concurrent metastatic disease was found in 28 patients, the majority of which was present in the lung (86\%), followed by metastases to the brain (25\%), and bone (21\%).

\section{Management and outcomes}

Surgical intervention was used in 20 of the 48 cases identified, half of which had no other concurrent metastatic disease. Four patients underwent total gastrectomy. The remainder had partial gastrectomies including 5 subtotal gastrectomies, 4 wedge resections and 1 antrectomy. Six cases did not specify the type of surgery. Three of the cases reporting use of wedge resections were performed for treatment of lesions $<7$ centimetres located in the gastric body.
Of those patients who did not receive surgery, 10 had endoscopic therapy, either in the form of polypectomy, ablation or mucosal resection. Palliative embolization was the treatment of choice in 2 cases, both requiring multiple embolizations in order to achieve haemostasis. Lamb et al. (2005) report a case of a patient who required 6 embolizations following 10 upper GI bleeds. Eight patients received chemotherapy and 7 did not receive any treatment. There were no case reports in the literature which described the use of palliative radiotherapy.

Cessation of bleeding was achieved in all patients who underwent surgical or endoscopic treatment for bleeding lesions (Figure 3).

\section{Survival}

Survival rates are generally poor with metastatic RCC, with most patients dying a few months after diagnosis [1,2]. In those with metastases to other organs in addition to the stomach, outcomes were worse (range 4 weeks to 36 months survival) than in those with isolated gastric metastases (range 4 weeks survival to alive after 6 years). Nearly half of the cases with widespread metastatic disease did not survive beyond 6 months, although several papers did not report survival data.

Of the 10 patients who received surgical intervention for isolated gastric metastases, 5 were cancer-free after 2-18 months. As only 7 cases in this category had published survival statistics, it is evident that surgical intervention carries symptomatic benefit in these patients, as well as the potential for curative treatment.

Overall survival for patients with RCC metastases to the stomach ranged from 4 weeks to alive after 6 years.

\section{Discussion}

Gastric metastases from RCC are rare and usually present with significant upper gastrointestinal haemorrhage.

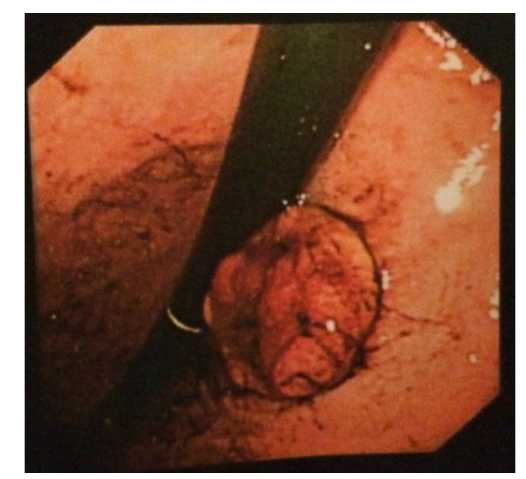

Figure 1: Ms DA - nodular gastric metastasis with mucosal ulceration.

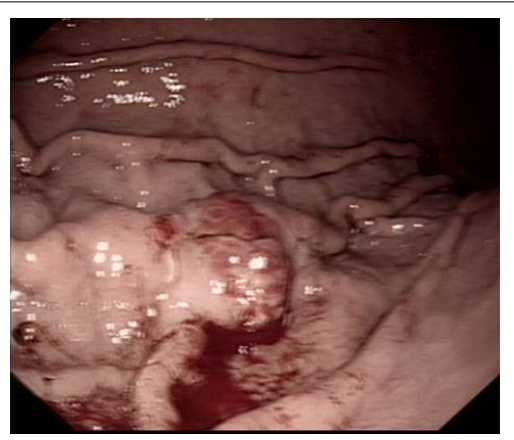

Figure 2: Mrs HB - bleeding mass with overlying mucosal ulceration in the greater curvature of the stomach. 
Table I: Case Reports of Gastric Metastases in RCC.

\begin{tabular}{|c|c|c|c|c|c|c|c|c|c|c|c|c|}
\hline Paper & Year & $\begin{array}{c}\text { Age } \\
\text { (years) }\end{array}$ & Sex & $\begin{array}{l}\text { Presenting } \\
\text { Complaint }\end{array}$ & $\begin{array}{c}\text { Interval Post- } \\
\text { Nephrectomy } \\
\text { (years) }\end{array}$ & Location & $\begin{array}{l}\text { Number of } \\
\text { lesions }\end{array}$ & $\begin{array}{l}\text { Type of } \\
\text { Lesion }\end{array}$ & Treatment & $\begin{array}{c}\text { Other } \\
\text { Metastases }\end{array}$ & $\begin{array}{l}\text { Survival } \\
\text { outcomes }\end{array}$ & Ref. \\
\hline Sullivan et al. & 1980 & 69 & $M$ & Melaena & 7.5 & Antrum & Single & Polypoid & Antrectomy & None & - & [17] \\
\hline Bisesti et al. & 1984 & 64 & M & Chest pain & 14 & Antrum & Single & Ulcer & Subtotal Gx & None & - & [18] \\
\hline Nakamura et al. & 1984 & 65 & M & Melaena & 9 & - & - & - & Partial Gx & lleum & $\begin{array}{l}\text { Died } 33 \text { days } \\
\text { post-op }\end{array}$ & [19] \\
\hline Ibáñez Olcoz et al. & 1989 & 60 & $\mathrm{~F}$ & Melaena & 1.8 & Body & Multiple & Polypoid & None & Lung, brain & - & [20] \\
\hline Márquez et al. & 1992 & 70 & M & Melaena & 0.1 & Body & Single & Ulcer & None & Lung & $\begin{array}{l}\text { Died after } 4 \\
\text { weeks }\end{array}$ & [21] \\
\hline Durous et al. & 1992 & 66 & M & Anaemia & 12 & Fundus & Multiple & - & Interferon & Lung, parotid & - & [22] \\
\hline Otowa et al. & 1992 & 61 & $\mathrm{~F}$ & Haematemesis & 0 & Body & Multiple & - & Total Gx & None & $\begin{array}{l}\text { Died } 3 \text { months } \\
\text { post-op }\end{array}$ & [23] \\
\hline Herrera Puerto et al. & 1993 & 63 & M & Haematemesis & 0.1 & Antrum & Single & Ulcer & None & None & $\begin{array}{c}\text { Died } 4 \\
\text { weeks post } \\
\text { nephrectomy }\end{array}$ & [24] \\
\hline Boruchowicz et al. & 1995 & 48 & M & Dysphagia & 1.3 & Fundus & Single & Polypoid & Chemotherapy & $\begin{array}{l}\text { Lung, liver, } \\
\text { oesophagus }\end{array}$ & - & [25] \\
\hline Blake et al. & 1995 & 63 & M & Haematemesis & 6 & - & Single & Tumour & $\begin{array}{c}\text { Palliative } \\
\text { embolization }\end{array}$ & Lung & $\begin{array}{l}\text { Alive after } 5 \\
\text { months }\end{array}$ & [26] \\
\hline Odori et al. & 1998 & 59 & M & Asymptomatic & 4.4 & Body & Single & Ulcer & Total Gx & None & $\begin{array}{l}\text { No tumour } \\
\text { recurrence at } \\
17 \text { months }\end{array}$ & [27] \\
\hline Picchio et al. & 2000 & 50 & $\mathrm{~F}$ & Melaena & 14 & Body & Single & Polypoid & Subtotal Gx & None & $\begin{array}{l}\text { No tumour } \\
\text { recurrence at } 6 \\
\text { months }\end{array}$ & [28] \\
\hline Mascarenhas et al. & 2001 & 66 & M & Haematemesis & 7 & Body & Single & Ulcer & Partial Gx & Lung, pleura & $\begin{array}{l}\text { Died after } 36 \\
\text { months }\end{array}$ & [29] \\
\hline Suárez-Ortega et al. & 2004 & 70 & $\mathrm{~F}$ & Melaena & 0 & - & Multiple & Polypoid & Palliative & Lung & - & [30] \\
\hline Kobayashi et al. & 2004 & 78 & $M$ & Anaemia & 6.2 & Body & Single & Not stated & Gx (NOS) & None & $\begin{array}{l}\text { Died after } 5 \\
\text { months }\end{array}$ & [31] \\
\hline Kok et al. & 2004 & 60 & M & Melaena & 20 & Body & Multiple & Tumour & - & - & - & [32] \\
\hline Suárez Fonseca et al. & 2004 & 61 & $\mathrm{~F}$ & Melaena & 4 & Body & Single & Polypoid & Palliative & Lung & - & [33] \\
\hline Lamb et al. & 2005 & 69 & $\mathrm{~F}$ & Haematemesis & 3 & Body & Single & Tumour & $\begin{array}{c}\text { Palliative } \\
\text { embolization } \\
\text { (x6) }\end{array}$ & Lung & $\begin{array}{l}\text { Died after } 23 \\
\text { months }\end{array}$ & [34] \\
\hline Portanova et al. & 2006 & 67 & $\mathrm{~F}$ & Melaena & 5 & Body & Single & Polypoid & Total Gx & Pancreas & $\begin{array}{c}\text { Alive after } 2 \\
\text { weeks }\end{array}$ & [35] \\
\hline Hollerbach et al. & 2006 & 56 & M & Anaemia & - & Body & Multiple & Polypoid & $\begin{array}{l}\text { Endoscopic } \\
\text { mucosal } \\
\text { resection }\end{array}$ & None & - & [36] \\
\hline Riviello et al. & 2006 & 68 & M & Melaena & 11 & Fundus & Single & Polypoid & $\begin{array}{c}\text { Total Gx, } \\
\text { chemotherapy }\end{array}$ & $\begin{array}{l}\text { Lung, spleen, } \\
\text { pancreas, } \\
\text { liver, lymph } \\
\text { nodes }\end{array}$ & $\begin{array}{c}\text { Died after } 2 \\
\text { years }\end{array}$ & [37] \\
\hline Saidi et al. & 2007 & - & - & Melaena & 10 & Body & Single & Polypoid & Wedge Rx & None & $\begin{array}{c}\text { Disease free } \\
\text { after } 18 \text { months }\end{array}$ & [38] \\
\hline Pezzoli et al. & 2007 & 78 & M & Anaemia & 5 & Body & Multiple & Polypoid & $\begin{array}{l}\text { Electrosurgical } \\
\text { snare resection }\end{array}$ & - & $\begin{array}{l}\text { Died after } 6 \\
\text { months }\end{array}$ & [3] \\
\hline Haffner et al. & 2007 & 80 & M & Anaemia & 0 & Fundus & Multiple & Ulcer & \begin{tabular}{|c|}
$\begin{array}{c}\text { Endoscopic } \\
\text { ablation }\end{array}$ \\
\end{tabular} & Lung & $\begin{array}{l}\text { Alive after } 5 \\
\text { months }\end{array}$ & [39] \\
\hline Ko et al. & 2008 & 71 & M & $\begin{array}{l}\text { Abdominal } \\
\text { mass }\end{array}$ & - & Body & Multiple & Tumour & - & Lung & - & [40] \\
\hline Roh et al. & 2008 & 60 & $\mathrm{~F}$ & Dyspepsia & 8 & Body & Multiple & Polypoid & Subtotal Gx & None & - & [41] \\
\hline Pollheimer et al. & 2008 & 69 & M & Abdominal pain & 4.2 & Body & Single & Ulcer & Tamoxifen & $\begin{array}{l}\text { Lung, bone, } \\
\text { adrenal }\end{array}$ & $\begin{array}{l}\text { Died after } 19 \\
\text { months }\end{array}$ & [5] \\
\hline Pollheimer et al. & 2008 & 77 & M & Asymptomatic & 6.3 & Antrum & Single & Ulcer & Interferon & Lung, bone & $\begin{array}{l}\text { Died after } 4 \\
\text { months }\end{array}$ & [5] \\
\hline Pollheimer et al. & 2008 & 83 & $\mathrm{~F}$ & Melaena & 1.7 & Antrum & Multiple & - & $\begin{array}{l}\text { Endoscopic } \\
\text { ablation, } \\
\text { Interferon }\end{array}$ & $\begin{array}{l}\text { Lung, liver, } \\
\text { pancreas }\end{array}$ & $\begin{array}{l}\text { Died after } 5 \\
\text { months }\end{array}$ & [5] \\
\hline Pollheimer et al. & 2008 & 65 & $\mathrm{~F}$ & $\begin{array}{c}\text { Haematemesis } \\
\text { \& Melaena }\end{array}$ & 13.1 & - & Multiple & - & $\begin{array}{l}\text { Endoscopic } \\
\text { ablation }\end{array}$ & Lung, brain & $\begin{array}{l}\text { Died after } 3 \\
\text { months }\end{array}$ & [5] \\
\hline Pollheimer et al. & 2008 & 69 & M & Abdominal pain & 9.3 & Body & Multiple & - & $\begin{array}{l}\text { Endoscopic } \\
\text { ablation, } \\
\text { Sunitinib }\end{array}$ & Lung, bone & $\begin{array}{c}\text { Alive after } 2 \\
\text { years }\end{array}$ & [5] \\
\hline Maeda et al. & 2009 & 49 & $M$ & Anaemia & 1.7 & Body & Single & Polypoid & Partial Gx & - & - & [42] \\
\hline Kibria et al. & 2009 & 53 & M & Melaena & 0 & Fundus & Single & Polypoid & None & Lung, bone & $\begin{array}{c}\text { Died after } 2 \\
\text { months }\end{array}$ & [8] \\
\hline Yamamoto et al. & 2009 & 74 & M & Melaena & 5 & Body & Single & Polypoid & Wedge $R x$ & Brain & $\begin{array}{l}\text { Died } 1 \text { month } \\
\text { post-op }\end{array}$ & {$[4]$} \\
\hline
\end{tabular}




\begin{tabular}{|c|c|c|c|c|c|c|c|c|c|c|c|c|}
\hline Sugasawa et al. & 2010 & 69 & M & Melaena & 19 & Fundus & Single & Ulcer & Wedge Rx & None & \begin{tabular}{|c|} 
Disease free \\
after 12 months
\end{tabular} & [43] \\
\hline Tiwari et al. & 2010 & 58 & $\mathrm{~F}$ & $\begin{array}{c}\text { Haematemesis } \\
\text { \& Melaena }\end{array}$ & 0 & Antrum & Single & Polypoid & Subtotal Gx & Lung & $\begin{array}{l}\text { Died } 2 \text { months } \\
\text { post-op }\end{array}$ & [7] \\
\hline Palade et al. & 2011 & - & - & Melaena & 8 & - & Single & Ulcer & Partial Gx & $\begin{array}{l}\text { Lung, brain, } \\
\text { bone }\end{array}$ & - & [44] \\
\hline Cruz et al. & 2011 & 56 & $\mathrm{~F}$ & Melaena & 6 & Antrum & Single & Tumour & Subtotal Gx & Lung, brain & - & [45] \\
\hline Eslick et al. & 2011 & 65 & M & PR Bleeding & 9 & Body & Single & Ulcer & $\begin{array}{l}\text { Endoscopic } \\
\text { polypectomy }\end{array}$ & None & $\begin{array}{l}\text { Alive after } 6 \\
\text { years }\end{array}$ & [2] \\
\hline Rodrigues et al. & 2012 & 45 & $\mathrm{~F}$ & Haematemesis & 9 & Body & Single & Ulcer & Sunitinib & Lung, ovary & $\begin{array}{l}\text { Died after } 4 \\
\text { months }\end{array}$ & [46] \\
\hline Namikawa et al. & 2012 & 65 & M & Mass on CT & 23 & Body & Single & Polypoid & Wedge Rx & None & $\begin{array}{l}\text { Disease free } \\
\text { after } 2 \text { months }\end{array}$ & [9] \\
\hline $\begin{array}{c}\text { Gómez-de-la-Cuesta } \\
\text { et al. }\end{array}$ & 2012 & 87 & $\mathrm{~F}$ & Melaena & 4 & Body & Multiple & Polypoid & Palliative & $\begin{array}{l}\text { Lung, } \\
\text { pancreas }\end{array}$ & - & [10] \\
\hline Siriwardana et al. & 2012 & 71 & M & Anaemia & 3 & - & Single & Polypoid & $\begin{array}{l}\text { Endoscopic } \\
\text { mucosal } \\
\text { resection }\end{array}$ & None & $\begin{array}{c}\text { Disease free } \\
\text { after } 15 \text { months }\end{array}$ & [47] \\
\hline Kim et al. & 2012 & 79 & M & Abdominal pain & 0 & Body & Single & Ulcer & $\begin{array}{l}\text { Endoscopic } \\
\text { submucosal } \\
\text { dissection }\end{array}$ & None & $\begin{array}{l}\text { Disease free } \\
\text { after } 6 \text { months }\end{array}$ & [6] \\
\hline Thoufeeq et al. & 2012 & 59 & $\mathrm{~F}$ & Dyspepsia & 3 & Fundus & Single & Polypoid & Sunitinib & Brain & - & [48] \\
\hline Onorati et al. & 2013 & 80 & - & - & 20 & - & - & - & - & - & - & [49] \\
\hline Sakurai et al. & 2014 & 61 & M & Melaena & 2 & Body & Single & Polypoid & Partial Gx & $\begin{array}{l}\text { Lung, bone, } \\
\text { brain }\end{array}$ & $\begin{array}{l}\text { Died } 4 \text { months } \\
\text { post-op }\end{array}$ & [50] \\
\hline Ikari et al. & 2014 & 64 & M & - & 22 & - & Single & Tumour & $\begin{array}{l}\text { Endoscopic } \\
\text { submucosal } \\
\text { dissection }\end{array}$ & None & $\begin{array}{c}\text { Disease free } \\
\text { after } 30 \text { months }\end{array}$ & [51] \\
\hline
\end{tabular}

\section{Treatment Modalities}
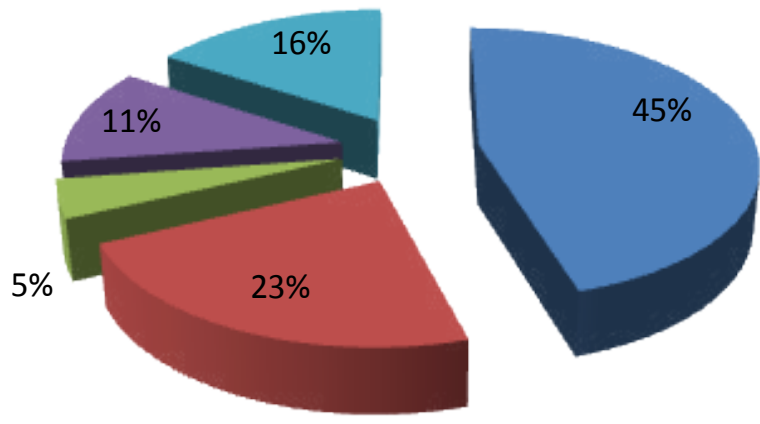

- Surgery

Endoscopic Therapy

- Palliative Embolisation

Chemotherapy

No Treatment/Palliative

Figure 3: Treatment Modalities for Management of Gastric Metastases in RCC $(n=44)$.

Establishing the histopathological differentiation between metastatic disease and other tumours such as primary gastrointestinal stromal tumours (GIST) is essential in determining the appropriate treatment [9].

Laparoscopic wedge resection is the treatment of choice for isolated small/medium gastric tumours $(<7$ centimetres) near the greater curvature of the stomach as it is associated with quicker recovery in comparison to open procedures [10]. Saidi et al. (2007) report one case where this resection technique was used in a patient with an isolated gastric metastasis of RCC origin, after which they remained diseasefree 18 months following surgery. Our patient is one of the longest surviving patients after laparoscopic wedge resection for isolated gastric RCC metastasis and remains disease-free 8 years post-surgery.

Subtotal and total gastrectomies are more frequently reported and are used to treat larger tumours or those which are localised within the antral or fundal regions of the stomach $[2,11]$. In the cases reviewed, all patients who underwent wedge resection in the absence of metastases to other organs were disease-free after 2-18 months with no evidence of further bleeding.

Endoscopic clipping is used to achieve haemostasis in upper GI bleeding, although it is also a technique used to localize gastric or oesophageal tumours to aid external beam radiotherapy [12]. Radiotherapy to gastric tumours is primarily utilised to palliatively treat symptoms of bleeding, pain and dysphagia $[13,14]$. To the best of our knowledge, there are no case reports on gastric metastases from renal carcinoma in which radiotherapy has been used as a treatment modality for cessation of bleeding.

Given the relatively small number of patients who develop gastric metastases from RCC, it would not be feasible to conduct trials to determine which interventions have the best outcomes. Hence, based on our experience and our literature review, we propose the following paradigm for treating gastric metastases from RCC primary (Figure 4) 


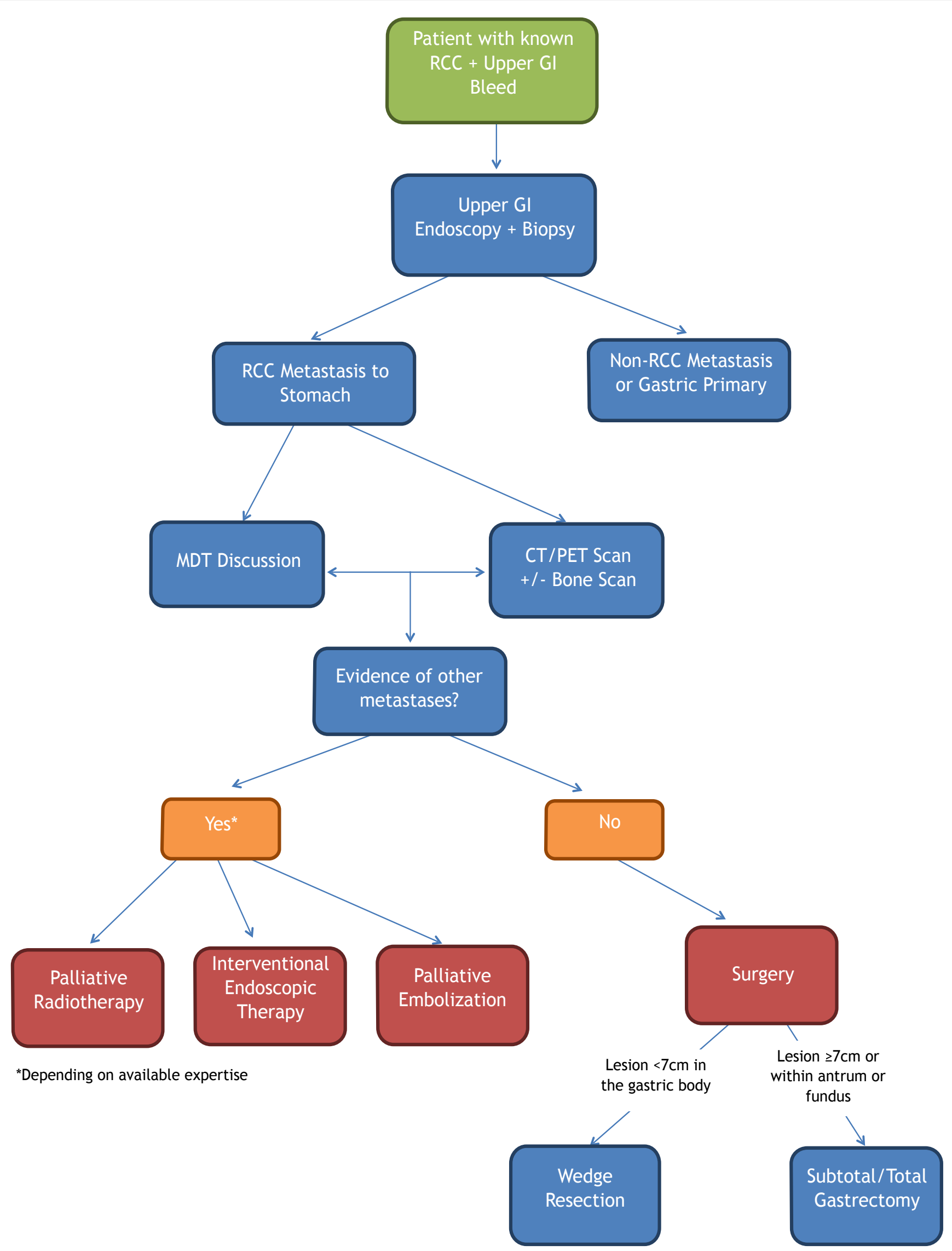

RCC: Renal Cell Carcinoma; MDT: Multidisciplinary Team; CT: Computed Tomography.

Figure 4: Treatment Paradigm for Management of Bleeding Gastric RCC Metastases. 
Discussion of such cases in a multi-disciplinary setting is critical. It is thought that oncology patients who are discussed at such meetings often have better outcomes $[15,16]$.

\section{Conclusion}

Gastric metastases in RCC are uncommon, but not as rare as once thought. They can cause significant haemorrhage and are generally associated with poor prognosis. Treatment should be patient-tailored depending on general condition at time of presentation, presence of extra-gastric metastases and the available resources and expertise. However, based on both our experience and the literature, we suggest that surgical intervention has good therapeutic and prognostic benefit in patients with isolated metastatic disease to the stomach. On the other hand, for those with widespread metastatic disease, other management options, if available, including embolization therapy, endoscopic submucosal resection and chemotherapy, should be considered. Furthermore, we advise that radiotherapy should also be considered as a viable option in the management of patients with bleeding lesions and concurrent metastases.

\section{Conflicts of interest}

The authors declare that they have no conflicts of interest.

\section{Reference}

1. WHO (2008) World Cancer Report 2008. International Agency for Research on Cancer 438-443.

2. Kim MY, Jung HY, Choi KD, Song HJ, Lee JH, et al. (2012) Solitary synchronous metastatic gastric cancer arising from $\mathrm{t} 1 \mathrm{~b}$ renal cell carcinoma: a case report and systematic review. Gut Liver 6: 388-394.

3. Eslick GD, Kalantar JS (2011) Gastric metastasis in renal cell carcinoma: a case report and systematic review. J Gastrointest Cancer 42: 296-301.

4. Pezzoli A, Matarese V, Boccia S, Simone L, Gullini S (2007) Gastrointestinal bleeding from gastric metastasis of renal cell carcinoma, treated by endoscopic polypectomy. Endoscopy 39 Suppl 1: E52.

5. Yamamoto D, Hamada Y, Okazaki S, Kawakami K, Kanzaki S, et al. (2009) Metastatic gastric tumor from renal cell carcinoma. Gastric Cancer 12: 170-173.

6. Pollheimer MJ, Hinterleitner TA, Pollheimer VS, Schlemmer A, Langner C (2008) Renal cell carcinoma metastatic to the stomach: single-centre experience and literature review. BJU Int 102: 315-319.

7. Tiwari P, Tiwari A, Vijay M, Kumar S, Kundu AK (2010) Upper gastro-intestinal bleeding - Rare presentation of renal cell carcinoma. Urol Ann 2: 127-129.

8. Kibria R, Sharma K, Ali SA, Rao P (2009) Upper gastrointestinal bleeding revealing the stomach metastases of renal cell carcinoma. J Gastrointest Cancer 40: 51-54.

9. Namikawa T, Hanazaki K (2014) Clinicopathological features and treatment outcomes of metastatic tumors in the stomach. Surg Today 44: 1392-1399.

10. Gómez-de-la-Cuesta S, Fernández-Salazar L, Velayos-Jiménez B, MachoConesa A, Ruiz-Rebollo L, et al. (2012) Gastric metastasis from renal cell carcinoma. Rev Esp Enferm Dig 104: 334-335.

11. Bucher P, Egger JF, Gervaz P, Ris F, Weintraub D, et al. (2006) An audit of surgical management of gastrointestinal stromal tumours (GIST). Eur J Surg Oncol 32: 310-314

12. Romagnuolo J (2009) Endoscopic clips: past, present and future. Can J Gastroenterol 23: 158-160.

13. McCloskey SA, Yang GY (2009) Benefits and challenges of radiation therapy in gastric cancer: techniques for improving outcomes. Gastrointest Cancer Res 3: $15-19$

14. Buergy D, Lohr F, Baack T, Siebenlist K, Haneder S, et al. (2012) Radiotherapy for tumors of the stomach and gastroesophageal junction--a review of its role in multimodal therapy. Radiat Oncol 7: 192.

15. Chirgwin J, Craike M, Gray C, Watty K, Mileshkin L, et al. (2010) Does multidisciplinary care enhance the management of advanced breast cancer? evaluation of advanced breast cancer multidisciplinary team meetings. J Oncol Pract 6: 294-300.

16. Perri F, Muto P, Aversa C, Daponte A, Della Vittoria G, et al. (2013) Integrated therapeutic approaches in head and neck cancer: the importance of multidisciplinary team management. Anticancer Agents Med Chem 13: 834-843.
17. Sullivan WG, Cabot EB, Donohue RE (1980) Metastatic renal cell carcinoma to stomach. Urology 15: 375-378.

18. Bisesti V, Parrella E, Cataldi C, Rizzo R, Onufrio A (1984) Ser di un raro caso di metastasi gastrica di adenocarcinoma renale. Osp Ital Pediatr 19: 609-620.

19. Nakamura R, Shimada A, Hara Y, Hirasawa M, Ando H et al. (1984) One case of intussusception due to intestinal metastasis of renal cell carcinoma. Jpn J Clin Surg 43: 1637-1640.

20. Ibáñez Olcoz J, Jiménez López CE, Oteo Revuelta JA, Cobo Huici F, Sara Ongay MJ, et al. (1989) [Gastric metastasis of renal adenocarcinoma. Presentation of a case and review of the literature]. Rev Esp Enferm Apar Dig 76: 259-261.

21. Márquez JL, Herrera JM, Herrera J, Caballero M, Narváez I, et al. (1992) [Gastric metastasis of renal cell adenocarcinoma]. Rev Esp Enferm Dig 81 129-130.

22. Durous E, Isaac S, Dubreuil C, Braillon G, Bonvoisin S, et al. (1992) [Late gastric metastasis of cancer of the kidney]. Presse Med 21: 996.

23. Otowa T, Muto I (1992) A case of synchronous gastric metastasis from rena cell carcinoma with the chief complaint of haematemesis. Jpn J Clin Surg 53: 1219-1222.

24. Herrera Puerto J, Caballero Gómez M, Márquez Galán JL, Domínguez Bravo C Soler Fernández JL, et al. (1993) [Metastatic hypernephroma of the stomach] Arch Esp Urol 46: 729-731.

25. Boruchowicz A, Desreumaux P, Maunoury V, Colombel JF (1995) Dysphagia revealing esophageal and gastric metastases of renal carcinoma. Am J Gastroenterol 90: 2263-2264.

26. Blake MA, Owens A, O'Donoghue DP, MacErlean DP (1995) Embolotherapy for massive upper gastrointestinal haemorrhage secondary to metastatic renal cell carcinoma: report of three cases. Gut 37: 835-837.

27. Odori T, Tsuboi Y, Katoh K, Yamada K, Morita K, et al. (1998) A solitary hematogenous metastasis to the gastric wall from renal cell carcinoma four years after radical nephrectomy. J Clin Gastroenterol 26: 153-154.

28. Picchio M, Paioletti A, Santini E, lacoponi S, Cordahi M (2000) Gastric metastasis from renal cell carcinoma fourteen years after radical nephrectomy. Acta Chir Belg 100: 228-230.

29. Mascarenhas B, Konety B, Rubin JT (2001) Recurrent metastatic renal cell carcinoma presenting as a bleeding gastric ulcer after a complete response to high-dose interleukin-2 treatment. Urology 57: 168.

30. Suárez-Ortega S, Limeres-González MA, Rodríguez-Fernández JM, Rivero JC, Santamaría P et al. (2004) Digestive bleeding as the clinical presentation of disseminated renal cell carcinoma. Rev Esp Geriatr Gerontol 39: 209-211.

31. Kobayashi O, Murakami H, Yoshida T, Cho H, Yoshikawa T, et al. (2004) Clinical diagnosis of metastatic gastric tumors: clinicopathologic findings and prognosis of nine patients in a single cancer center. World J Surg 28: 548-551.

32. Kok Wee L, Shyu RY, Sheu LF, Hsieh TY, Yan JC, et al. (2004) Metastatic renal cell cancer. Gastrointest Endosc 60: 265.

33. Suárez Fonseca C, Carballido Rodríguez J, González Lama Y, Sola Galarza I, Rodríguez Reina G, et al. (2004) Gastric metastasis from renal cell carcinoma. Pathogenical hypothesis and literature revision. Actas Urol Esp 286: 472-476.

34. Lamb GW, Moss J, Edwards R, Aitchison M (2005) Case report: octreotide as an adjunct to embolisation in the management of recurrent bleeding upper gastrointestinal metastases from primary renal cell cancer. Int Urol Nephrol 37: 691-693.

35. Portanova M, Yabar A, Lombardi E, Vargas F, Mena V, et al. (2006) [Concomitant gastric and pancreatic metastases from renal cell carcinoma: case study]. Rev Gastroenterol Peru 26: 84-88.

36. Hollerbach S, Freund U, Stolte M (2006) Recurrent upper-gastrointestina bleeding from multiple gastric polyps treated successfully by endoscopic mucosal resection. Clin Gastroenterol Hepatol 4: xxxii.

37. Riviello C, Tanini I, Cipriani G, Pantaleo P, Nozzoli C, et al. (2006) Unusual gastric and pancreatic metastatic renal cell carcinoma presentation 10 years after surgery and immunotherapy: A case report and a review of literature. World J Gastroenterol 12: 5234-5236.

38. Saidi RF, Remine SG (2007) Isolated gastric metastasis from renal cell carcinoma 10 years after radical nephrectomy. J Gastroenterol Hepatol 22 143-144.

39. Haffner J, Morel JF, Maunoury V, Caty A, Biserte J, et al. (2007) [Gastric or duodenal metastases from clear cell renal cell carcinoma. Report of two cases and review of the literature]. Prog Urol 17: 1305-1309. 
40. Ko WO, Yang US, Kim NS, An JK, Kim KJ et al. (2008) A case of gastric metastasis of a renal cell carcinoma. Korean J Gastrointest Endosc. 36: 288291.

41. Roh JH, Kim HJ, Jang KT, Kim KM, Kim JJ, et al. (2008) Metastatic renal cell carcinoma masquerading as a primary gastric carcinoma associated with synchronous early gastric carcinoma. Pathology 40: 83-85.

42. Maeda T, Kozakai N, Nishiyama T, Ishii T, Sugiura H, et al. (2009) [Gastric metastasis from renal cell carcinoma 20 months after radical nephrectomy: a case report]. Hinyokika Kiyo 55: 137-140.

43. Sugasawa H, Ichikura T, Ono S, Tsujimoto H, Hiraki S, et al. (2010) Isolated gastric metastasis from renal cell carcinoma 19 years after radical nephrectomy. Int J Clin Oncol 15: 196-200.

44. Palade R, Voiculescu D, Suliman E, Simion G (2011) [Gastric metastasis of clear cell renal carcinoma]. Chirurgia (Bucur) 106: 379-382.

45. Cruz A, Ramírez LM, Sánchez E, Ruiz M, Moreno I, et al. (2011) Gastric metastasis from renal cancer six years after nephrectomy. Rev Esp Enferm Dig 103: 660-661.
46. Rodrigues S, Bastos $P$, Macedo $G$ (2012) A rare cause of hematemesis: gastric metastases from renal cell carcinoma. Gastrointest Endosc 75: 894.

47. Siriwardana HP, Harvey MH, Kadirkamanathan SS, Tang B, Kamel D, et al (2012) Endoscopic mucosal resection of a solitary metastatic tumor in the stomach: a case report. Surg Laparosc Endosc Percutan Tech 22: e132-e134.

48. Thoufeeq MH, Maleki N, Jagirdar N, Rembacken B, Jennings J (2012) Renal cell cancer diagnosed at endoscopy. Case Rep Gastrointest Med 2012: 360560

49. Onorati M, Petracco G2, Uboldi P2, Redaelli DG3, Romagnoli S4, et al. (2013) A solitary polypoid gastric metastasis 20 years after renal cell carcinoma: an event to be considered, and a brief review of the literature. Pathologica 105 132-136.

50. Sakurai K, Muguruma K, Yamazoe S, Kimura K, Toyokawa T, et al. (2014) Gastric metastasis from renal cell carcinoma with gastrointestinal bleeding: a case report and review of the literature. Int Surg 99: 86-90.

51. Ikari N, Miura O, Takeo S, Okamoto F, Okazaki Y et al. (2014) Pancreatic and gastric metastases occurring a decade after nephrectomy for renal cell carcinoma. J Gastroenterol 111: 311-317. 